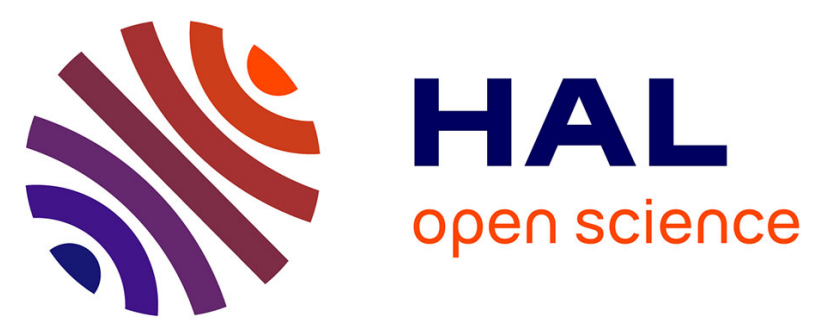

\title{
Effectiveness of teicoplanin versus vancomycin lock therapy in the treatment of port-related coagulase-negative staphylococci bacteraemia: a prospective case-series analysis
}

\author{
J.L. del Pozo, M. García Cenoz, S. Hernáez, A. Martínez, A. Serrera, A.
}

Aguinaga, M. Alonso, J. Leiva

\section{To cite this version:}

J.L. del Pozo, M. García Cenoz, S. Hernáez, A. Martínez, A. Serrera, et al.. Effectiveness of teicoplanin versus vancomycin lock therapy in the treatment of port-related coagulase-negative staphylococci bacteraemia: a prospective case-series analysis. International Journal of Antimicrobial Agents, 2009, 34 (5), pp.482. 10.1016/j.ijantimicag.2009.06.020 . hal-00556352

\section{HAL Id: hal-00556352 https://hal.science/hal-00556352}

Submitted on 16 Jan 2011

HAL is a multi-disciplinary open access archive for the deposit and dissemination of scientific research documents, whether they are published or not. The documents may come from teaching and research institutions in France or abroad, or from public or private research centers.
L'archive ouverte pluridisciplinaire HAL, est destinée au dépôt et à la diffusion de documents scientifiques de niveau recherche, publiés ou non, émanant des établissements d'enseignement et de recherche français ou étrangers, des laboratoires publics ou privés. 


\section{Accepted Manuscript}

Title: Effectiveness of teicoplanin versus vancomycin lock therapy in the treatment of port-related coagulase-negative staphylococci bacteraemia: a prospective case-series analysis

Authors: J.L. Del Pozo, M. García Cenoz, S. Hernáez, A. Martínez, A. Serrera, A. Aguinaga, M. Alonso, J. Leiva

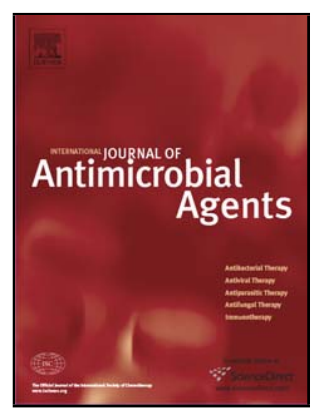

PII:

DOI:

Reference:

S0924-8579(09)00323-9

ANTAGE 3076

To appear in: International Journal of Antimicrobial Agents

Received date: $\quad$ 12-2-2009

Revised date: $\quad 14-4-2009$

Accepted date: $\quad$ 9-6-2009

Please cite this article as: Del Pozo JL, Cenoz MG, Hernáez S, Martínez A, Serrera A, Aguinaga A, Alonso M, Leiva J, Effectiveness of teicoplanin versus vancomycin lock therapy in the treatment of port-related coagulase-negative staphylococci bacteraemia: a prospective case-series analysis, International Journal of Antimicrobial Agents (2008), doi:10.1016/j.ijantimicag.2009.06.020

This is a PDF file of an unedited manuscript that has been accepted for publication. As a service to our customers we are providing this early version of the manuscript. The manuscript will undergo copyediting, typesetting, and review of the resulting proof before it is published in its final form. Please note that during the production process errors may be discovered which could affect the content, and all legal disclaimers that apply to the journal pertain. 


\section{Effectiveness of teicoplanin versus vancomycin lock therapy in the treatment of port-related coagulase-negative staphylococci bacteraemia: a prospective case-series analysis}

J.L. Del Pozo ${ }^{a, b, *}$, M. García Cenoz ${ }^{c}$, S. Hernáez ${ }^{b}, A$. Martínez $^{d}$, A. Serrera ${ }^{b}$, A. Aguinaga ${ }^{b}$, M. Alonso $^{b}$, J. Leiva ${ }^{b}$

a Division of Infectious Diseases, Clínica Universidad de Navarra, Pamplona, Spain

${ }^{\mathrm{b}}$ Department of Clinical Microbiology, Clínica Universidad de Navarra, Pamplona, Spain

${ }^{\mathrm{c}}$ Public Health Institute of Navarre, Pamplona, Spain

${ }^{d}$ Department of Radiology, Clínica Universidad de Navarra, Pamplona, Spain

ARTICLE INFO

Article history:

Received 12 February 2009

Accepted 9 June 2009

Keywords:

Catheter-related infection

Bacteraemia 


\section{Teicoplanin}

Biofilms

* Corresponding author. Tel.: +34 948255 400; fax: +34 948296500.

E-mail addresses: delpozo.jose@mayo.edu, jdelpozo@unav.es (J.L. Del Pozo). 


\section{ABSTRACT}

The aim of this study was to analyse the effectiveness of teicoplanin versus vancomycin lock therapy in the treatment of coagulase-negative staphylococci (CoNS) venous access port-related bloodstream infection (BSI). The study included 44 consecutive patients during a 36-month prospective case-series study. The primary endpoint was failure to cure. Treatment was successful in 39 patients. At the end of the study, the cumulative port survival rate was $100 \%$ in the teicoplanin lock group compared with $77 \%$ in the vancomycin lock group $(P=$ 0.06). In the Cox regression analysis, fever beyond $48 \mathrm{~h}$ of treatment was a significant predictor of treatment failure $(P=0.02)$. Use of vancomycin or teicoplanin locks had an effectiveness of $88.6 \%$ in the treatment of CoNS portrelated BSI. Teicoplanin locks reduced the failure rate from $18.5 \%$ to $0 \%$ compared with vancomycin locks. The presence of fever after the beginning antimicrobial lock therapy was associated with treatment failure. 


\section{Introduction}

Use of venous access ports has increased over the last decade and nowadays they are commonly used in the management of oncology patients. Port-related infections increase hospital-related costs and are a well recognised source of patient morbidity and mortality. Coagulase-negative staphylococci (CoNS) are the most common pathogen involved. Removal of a surgically implantable vascular device is often a management challenge and it is important to determine the role of conservative therapeutic approaches. Antibiotic lock therapy (ALT) is recommended in guidelines as treatment for patients with CoNS catheter-related bacteraemia [1-3]. Success rates have varied substantially among studies and, even in non-complicated patients, a 100\% rate has not been reached [4]. Vancomycin is considered the agent of choice to perform antimicrobial locks when staphylococci are involved. Teicoplanin is a glycopeptide with an antimicrobial spectrum similar to that of vancomycin that has shown better binding to siliconised polymers [5]. This phenomenon might be relevant in the effectiveness of antimicrobial locks to treat port-related bloodstream infections (BSIs) since silicone is the main inner surface port component. However, there is a lack of clinical research into this topic. The aim of this study was to compare the effectiveness of teicoplanin lock therapy versus vancomycin lock therapy in the treatment of CoNS venous access port-related BSI. 


\section{Patients and methods}

\subsection{Study location}

The study was carried out in a 350-bed university hospital in which ca. 300 venous access ports are implanted every year. All participants provided informed consent.

\subsection{Study design and patients}

An observational study was prospectively conducted including consecutive patients with port-related bacteraemia diagnosed at Clínica Universidad de Navarra (Pamplona, Spain). All hospitalised and non-hospitalised patients with a venous access port in place were eligible for inclusion. During the 36-month study period (1 June 2000 to 31 May 2003), consecutive patients meeting clinical criteria to suspect a port-related infection (fever $>38^{\circ} \mathrm{C}$ and no obvious site of infection other than the port) were recruited. Concomitant quantitative blood cultures were drawn through the device and percutaneously and were processed by the lysis-centrifugation method (Isolator system; Wampole Laboratories, Cranbury, NJ). Patients were diagnosed with a port-related BSI according to accepted criteria (i.e. cultures positive from both sites and the concentration of microorganisms in the culture from the port is three- to five-fold greater than in the peripherally drawn culture) $[6,7]$. Microbial isolates were identified using standard techniques, and susceptibility results were interpreted according to the recommendations of the Clinical and Laboratory Standards Institute [8]. 


\subsection{Exclusion criteria}

Patients were excluded from the study if they had one or more of the following criteria: isolation of a microorganism other than CoNS; polymicrobial infection; evidence of pocket or subcutaneous tract infection; unstable clinical status; existence of a metastatic complication (endocarditis, septic thrombophlebitis or osteomyelitis); and patient previously included in the study. Moreover, patients were also excluded when no peripheral vein or port blood was available.

\subsection{Treatment}

All included patients received ALT. Vancomycin was used as the lock solution during the first 20 months of study and teicoplanin was used during the last 16 months. Syringes $(5 \mathrm{~mL})$ were filled with vancomycin $(2 \mathrm{mg} / \mathrm{mL})$ or teicoplanin (10 $\mathrm{mg} / \mathrm{mL}$ ) plus heparin (to achieve a final concentration of $100 \mathrm{U} / \mathrm{mL}$ ). The antimicrobial locks were replaced daily. During treatment, ports remained locked whenever not in use ( $\geq 8 \mathrm{~h}$ a day). Administration and duration of systemic antimicrobial therapy was performed according to current guidelines [1]. A quantitative blood culture was withdrawn from the port 1 week after the end of treatment, and treatment was considered a failure if the culture was positive for the same phenotypic strain that caused the primary episode. Patient follow-up was for a minimum of 12 months or until port removal or patient death. The following baseline data were collected: age; gender; underlying disease; date of port insertion; neutrophil count (neutropenia defined as $<500$ cells $/ \mathrm{mm}^{3}$ ); and use of parenteral nutrition through the port. Following diagnosis, data regarding 
antimicrobial lock concentration, lock duration, use of systemic antimicrobial therapy and presence of fever during treatment were collected

\subsection{Port culture}

Ports were aseptically removed and cultured when no longer required or when a complication developed. The catheter tip and chamber were processed separately by a vortexing-sonication technique for quantitative bacterial culture.

\subsection{Endpoint}

The primary endpoint used in this study was failure to cure the episode of portrelated BSI. Treatment failure was defined as port removal during or after therapy owing to fever persistence (defined as fever beyond $72 \mathrm{~h}$ after initiating ALT) or positive blood or venous access port culture with the same phenotypic strain after the end of treatment. If at the time of patient death or port removal no relapse of related $\mathrm{BSI}$ had been observed, it was considered that the primary endpoint was not reached.

\subsection{Statistical analysis}

The baseline characteristics of the teicoplanin lock group and the vancomycin lock group were compared by the Yates' $\chi^{2}$ test, the Fisher exact test or the $T$ test, as appropriate. The Kaplan-Meier survival method was used to estimate the treatment outcome. The log-rank test was used to detect differences between the 
two treatment groups. Cox regression analysis was performed to assess the influence of baseline or treatment variables on treatment failure. Data were evaluated by SPSS 14.0 package program (SPSS Inc., Chicago, IL). A $P$-value of $<0.05$ was considered statistically significant.

\section{Results}

In total, 90 patients were diagnosed with port-related bacteraemia. However, 28 patients with port-related BSIs other than CoNS and 5 patients with polymicrobial BSIs were excluded. In addition, 13 patients were also excluded because of the presence of pocket or subcutaneous tract infection (10), unstable clinical status (2) or existence of a metastatic complication (1). Thus, 44 patients were diagnosed with CoNS port-related BSI and included in the study. Table 1 illustrates that both treatment groups were similar with regard to baseline conditions: sex ratio $(P=0.08)$; presence of neutropenia $(P=0.72)$; use of parenteral nutrition through the port in the last 3 months $(P=1)$; and mean time from port insertion to first bloodstream-related infection $(P=0.16)$. The only significant difference between the groups was that the mean age of the teicoplanin lock group patients was 12 years more than those in the vancomycin lock group (44.4 years vs. 56.7 years; $P=0.02$ ).

All of the isolates were susceptible to vancomycin and teicoplanin. Vancomycin $\mathrm{MIC}_{50}$ and $\mathrm{MIC}_{90}$ values (minimum inhibitory concentrations for $50 \%$ and $90 \%$ of the organisms, respectively) were $0.5 \mathrm{mg} / \mathrm{L}$ and $2 \mathrm{mg} / \mathrm{L}$ during the first period and 
$0.5 \mathrm{mg} / \mathrm{L}$ and $4 \mathrm{mg} / \mathrm{L}$ during the second period, with no statistical differences. Similarly, there were no differences in teicoplanin MICs, with $\mathrm{MIC}_{50}$ and $\mathrm{MIC}_{90}$ values of $1 \mathrm{mg} / \mathrm{L}$ and $8 \mathrm{mg} / \mathrm{L}$ during the first period and $0.5 \mathrm{mg} / \mathrm{L}$ and $8 \mathrm{mg} / \mathrm{L}$ during the second period. Overall, treatment was successful in $39(88.6 \%)$ of the 44 episodes. Cure was documented in $22 / 27$ patients $(81.5 \%)$ treated with vancomycin locks and in $17 / 17$ patients $(100 \%)$ treated with teicoplanin locks, with no statistically significant differences between the two groups (Fisher exact test, $P=0.13)$. Both the vancomycin locks and the teicoplanin locks were administered over a mean time of 10 days (interquartile range 10-14 days). Of the 27 patients in the vancomycin lock group, $11(40.7 \%)$ received an oral antimicrobial agent, $13(48.1 \%)$ received an intravenous (i.v.) glycopeptide and 3 $(11.1 \%)$ received an i.v. antimicrobial agent other than a glycopeptide. Of the 17 patients in the teicoplanin lock group, $8(47.1 \%)$ received an oral antimicrobial agent, $6(35.3 \%)$ received an i.v. glycopeptide and $3(17.6 \%)$ received an i.v. antimicrobial agent other than a glycopeptide. No statistical difference $(P=0.29)$ was found between the two groups regarding the different agents or administration routes used.

At the end of the study follow-up the total cumulative port survival rate was $85.8 \%(100 \%$ in the teicoplanin lock group patients compared with $77 \%$ in the vancomycin lock group) (Fig. 1). No significant differences were found in port lifespan between the two groups by the log-rank test $(P=0.06)$. In three of the five failures observed in the vancomycin lock group, the port had to be removed 
for persistent fever after $72 \mathrm{~h}$ of treatment. Two of the patients had a positive blood culture with the same phenotypic strain, and in the third patient no blood culture was obtained. All three port cultures were positive with the same strain previously isolated. The other two cases were considered failures because of relapsing bacteraemia with the same phenotypic strain 7 days and 210 days, respectively, after finishing treatment. Follow-up was complete for 39 (88.6\%) of the 44 patients. Two patients were lost to follow-up in the vancomycin lock group and three patients were lost to follow-up in the teicoplanin lock group. The median length of patient follow-up was 180 days (range 0-1204 days) in the vancomycin group, with five patients having $<30$ days follow-up, and 239 days (range 30-244 days) in the teicoplanin group, with one patient having $<30$ days follow-up.

To explore whether any variables were a predictor of treatment failure, age, gender, neutropenia, parenteral nutrition, time from port placement to infection, systemic or oral antimicrobial use, type and route of antimicrobial, antimicrobial used in the lock solution and fever during the first $48 \mathrm{~h}$ of treatment were included in a Cox model as time-dependent predictors. The only significant predictor of treatment failure was fever after $48 \mathrm{~h}$ of treatment $(P=0.02)$. Patients who were febrile during the first $48 \mathrm{~h}$ of treatment were at an increased failure risk (33.7 times greater) than those that become afebrile during the first 48 h. 


\section{Discussion}

To the best of our knowledge, this is the first study to compare vancomycin and teicoplanin as lock solutions for the treatment of CoNS port-related BSI. Moreover, this is the largest prospective study on the outcome of port-related CoNS BSI treated with ALT. The present study shows that in patients with a CoNS port-related BSI, the use of vancomycin or teicoplanin locks has an effectiveness of nearly $90 \%$. Moreover, this study shows that teicoplanin locks reduce the failure rate to cure the infection from $18.5 \%$ to $0 \%$ compared with vancomycin locks. Differences were not statistically significant, although the $P$ value was close to the level of significance $(P=0.06)$, possibly due the small size of the groups. Interestingly, the presence of fever beyond $48 \mathrm{~h}$ after beginning ALT was significantly associated with treatment failure. However, this finding is based on only three failure episodes.

The utility of antimicrobial locks in the treatment of long-term intravascular device-related BSIs has been suggested in a recent randomised, blinded, multicentre trial [9]. The overall cure rate in our study was $88.6 \%$, which is in accordance with previous reports on the use of antimicrobial locks for the treatment of CoNS catheter-related BSIs $[3,4]$. Vancomycin is the most frequent antimicrobial used, although teicoplanin has also been used but with irregular results [10-12]. The antibiotic-lock technique with teicoplanin was systematically used in 24 patients with Staphylococcus epidermidis catheter-related sepsis during long-term parenteral nutrition [10]. The teicoplanin-lock technique 
prevented short-term catheter removal, but a second infection occurred in five cases. In two studies [11,12], teicoplanin locks were used in Gram-positive catheter-related infections in children. All infections were treated successfully and no catheters had to be removed. Wilcox et al. [5] showed that binding of teicoplanin to siliconised polymers was, on average, four times greater than that of vancomycin. Carsenti-Etessi et al. [13] found that subinhibitory concentrations of teicoplanin decreased adherence of $S$. epidermidis to tissue culture plates, whereas vancomycin had no activity. Teicoplanin achieved a significantly greater reduction in the counts of $S$. epidermidis adhering to polyurethane and silicone catheters compared with vancomycin in an in vitro model [14]. In an experimental mouse model, Romano et al. [15] showed that S. epidermidis colonisation of teicoplanin-loaded catheters was inhibited. Lee et al. [16] showed that staphylococcal biofilms were completely sterile after exposure to vancomycin ( 5 $\mathrm{mg} / \mathrm{mL})$ for 5 days and teicoplanin $(5 \mathrm{mg} / \mathrm{mL}$ and $10 \mathrm{mg} / \mathrm{mL})$ for 7 days using an in vitro model. There is no current recommendation regarding the dosing of locking solutions; vancomycin concentrations of $0.05-83 \mathrm{mg} / \mathrm{mL}$ and teicoplanin concentrations of $4-133 \mathrm{mg} / \mathrm{mL}$ have been used. The different concentrations used in this study (i.e. vancomycin $2 \mathrm{mg} / \mathrm{mL}$ vs. teicoplanin $10 \mathrm{mg} / \mathrm{mL}$ ) might play a relevant role on our results.

Our study has several limitations. The relatively small number of patients included makes it difficult to generalise the results obtained to the general population. However, port-related BSIs have a really low incidence, making 
patient recruitment difficult. We have shown a non-significant trend in favour of teicoplanin locks, but a much larger study group is needed to determine whether this trend is 'real'. A clinical randomised trial would have been more suitable, and potential bias related to patient selection could have been avoided. We decided to include patients according to very strict criteria, which probably led to the exclusion of patients with real port-related BSIs but without fulfilling criteria; on other hand, all included patients certainly had a port-related BSI. Besides, genotype studies of the isolated microorganisms were not performed and relapsing bacteraemia was assumed on the basis of phenotypic tests. Therefore, we cannot guarantee whether relapses were real relapses or re-infections. On the other hand, all patients included in this study were evaluated by the same staff, which ensures homogeneity in the diagnosis and management of the included patients. Despite these limitations, we believe that the reported data may be useful and serve as a basis to design future randomised clinical trials.

In conclusion, the present study shows that the use of teicoplanin locks, in addition to systemic antimicrobials, increased the cure rate from $81.5 \%$ to $100 \%$ and the port survival rate from $77 \%$ to $100 \%$ compared with vancomycin locks in the treatment of CoNS port-related BSI. In our study, presence of fever beyond $48 \mathrm{~h}$ after starting treatment was associated with treatment failure. Further research into the effectiveness of ALT to treat CoNS port-related infections is warranted. 
Funding: None.

Competing interests: None declared.

Ethical approval: The Institutional Review Board of Clínica Universidad de Navarra (Pamplona, Spain) approved the study protocol. 


\section{References}

[1] Mermel LA, Farr BM, Sherertz RJ, Raad, II, O'Grady N, Harris JS, et al. Guidelines for the management of intravascular catheter-related infections. Clin Infect Dis 2001;32:1249-72.

[2] Bouza E, Burillo A, Muñoz P. Catheter-related infections: diagnosis and intravascular treatment. J Chemother 2001;13[Spec No. 1(1)]:224-33.

[3] Fortun J, Grill F, Martin-Davila P, Blazquez J, Tato M, Sanchez-Corral J, et al. Treatment of long-term intravascular catheter-related bacteraemia with antibiotic-lock therapy. J Antimicrob Chemother 2006;58:816-21.

[4] Fernandez-Hidalgo N, Almirante B, Calleja R, Ruiz I, Planes AM, Rodriguez $D$, et al. Antibiotic-lock therapy for long-term intravascular catheter-related bacteraemia: results of an open, non-comparative study. J Antimicrob Chemother 2006;57:1172-80.

[5] Wilcox MH, Winstanley TG, Spencer RC. Binding of teicoplanin and vancomycin to polymer surfaces. J Antimicrob Chemother 1994;33:431-41.

[6] Capdevila JA, Planes AM, Palomar M, Gasser I, Almirante B, Pahissa A, et al. Value of differential quantitative blood cultures in the diagnosis of catheterrelated sepsis. Eur J Clin Microbiol Infect Dis 1992;11:403-7.

[7] Safdar N, Fine JP, Maki DG. Meta-analysis: methods for diagnosing intravascular device-related bloodstream infection. Ann Intern Med 2005;142:451-66. 
[8] Clinical and Laboratory Standards Institute. Performance standards for antimicrobial disk susceptibility tests; approved standard. 10th ed. Wayne, PA: CLSI; 2009.

[9] Rijnders BJ, Van Wijngaerden E, Vandecasteele SJ, Stas M, Peetermans WE. Treatment of long-term intravascular catheter-related bacteraemia with antibiotic lock: randomized, placebo-controlled trial. J Antimicrob Chemother 2005;55:90-4.

[10] Guedon C, Nouvellon M, Lalaude O, Lerebours E. Efficacy of antibioticlock technique with teicoplanin in Staphylococcus epidermidis catheterrelated sepsis during long-term parenteral nutrition. JPEN J Parenter Enteral Nutr 2002;26:109-13.

[11] McCarthy A, Byrne M, Breathnach F, O'Meara A. "In-situ" teicoplanin for central venous catheter infection. Ir J Med Sci 1995;164:125-7.

[12] McCarthy A, Rao JS, Byrne M, Breatnach F, O'Meara CA. Central venous catheter infections treated with teicoplanin. Eur J Haematol 1998;62:15-7.

[13] Carsenti-Etesse H, Durant J, Entenza J, Mondain V, Pradier C, Bernard E, et al. Effects of subinhibitory concentrations of vancomycin and teicoplanin on adherence of staphylococci to tissue culture plates. Antimicrob Agents Chemother 1993;37:921-3.

[14] Kropec A, Huebner J, Wursthorn M, Daschner FD. In vitro activity of vancomycin and teicoplanin against Staphylococcus aureus and Staphylococcus epidermidis colonizing catheters. Eur J Clin Microbiol Infect Dis $1993 ; 12: 545-8$. 
[15] Romano G, Berti M, Goldstein BP, Borghi A. Efficacy of a central venous catheter (Hydrocath) loaded with teicoplanin in preventing subcutaneous staphylococcal infection in the mouse. Zentralbl Bakteriol 1993;279:426-33.

[16] Lee JY, Ko KS, Peck KR, Oh WS, Song JH. In vitro evaluation of the antibiotic lock technique (ALT) for the treatment of catheter-related infections caused by staphylococci. J Antimicrob Chemother 2006;57:1110-5. 
Fig. 1. Treatment effectiveness curves according to treatment group. 


\section{Table 1}

Characteristics of patients included in the study

\begin{tabular}{|c|c|c|}
\hline & $\begin{array}{l}\text { Patients with } \\
\text { vancomycin locks }(n= \\
27)\end{array}$ & $\begin{array}{l}\text { Patients with } \\
\text { teicoplanin locks }(n= \\
17)\end{array}$ \\
\hline Age [mean (S.D.)] (years) & $44.4(18.6)$ & $56.7(13.1)$ \\
\hline \multicolumn{3}{|l|}{$\operatorname{Sex}(n)$} \\
\hline Male (\%) & $9(33.3)$ & $11(64.7)$ \\
\hline Female (\%) & $18(67.7)$ & $6(35.3)$ \\
\hline $\begin{array}{l}\text { Time before infection [mean } \\
\text { (S.D.)] (days) }\end{array}$ & $338.5(265.3)$ & $226.2(238.8)$ \\
\hline $\begin{array}{l}\text { Solid/haematological neoplasia } \\
(n)\end{array}$ & $21 / 6$ & $16 / 1$ \\
\hline Neutropenia $[n(\%)]$ & $7(25.9)$ & $3(17.6)$ \\
\hline $\operatorname{TPN}[n(\%)]$ & $3(11.1)$ & $2(11.8)$ \\
\hline ALT [mean (S.D.)] (h/day) & $14.7(5.7)$ & $17.8(7)$ \\
\hline \multicolumn{3}{|l|}{ ALT duration (days) } \\
\hline Mean & 10 & 10 \\
\hline IQR & $10-14$ & $10-14$ \\
\hline Systemic antibiotic use $[n(\%)]$ & $16(59.3)$ & $9(52.9)$ \\
\hline i.v. glycopeptide $[n(\%)]$ & $13(48.1)$ & $6(35.3)$ \\
\hline $\begin{array}{l}\text { i.v. antimicrobial other than a } \\
\text { glycopeptide }[n(\%)]\end{array}$ & $3(11.1)$ & $3(17.6)$ \\
\hline Oral antimicrobial agent $[n(\%)]$ & $11(40.7)$ & $8(47.1)$ \\
\hline
\end{tabular}

S.D., standard deviation; TPN, total parenteral nutrition; ALT, antimicrobial lock therapy; IQR, interquartile range; i.v., intravenous. 


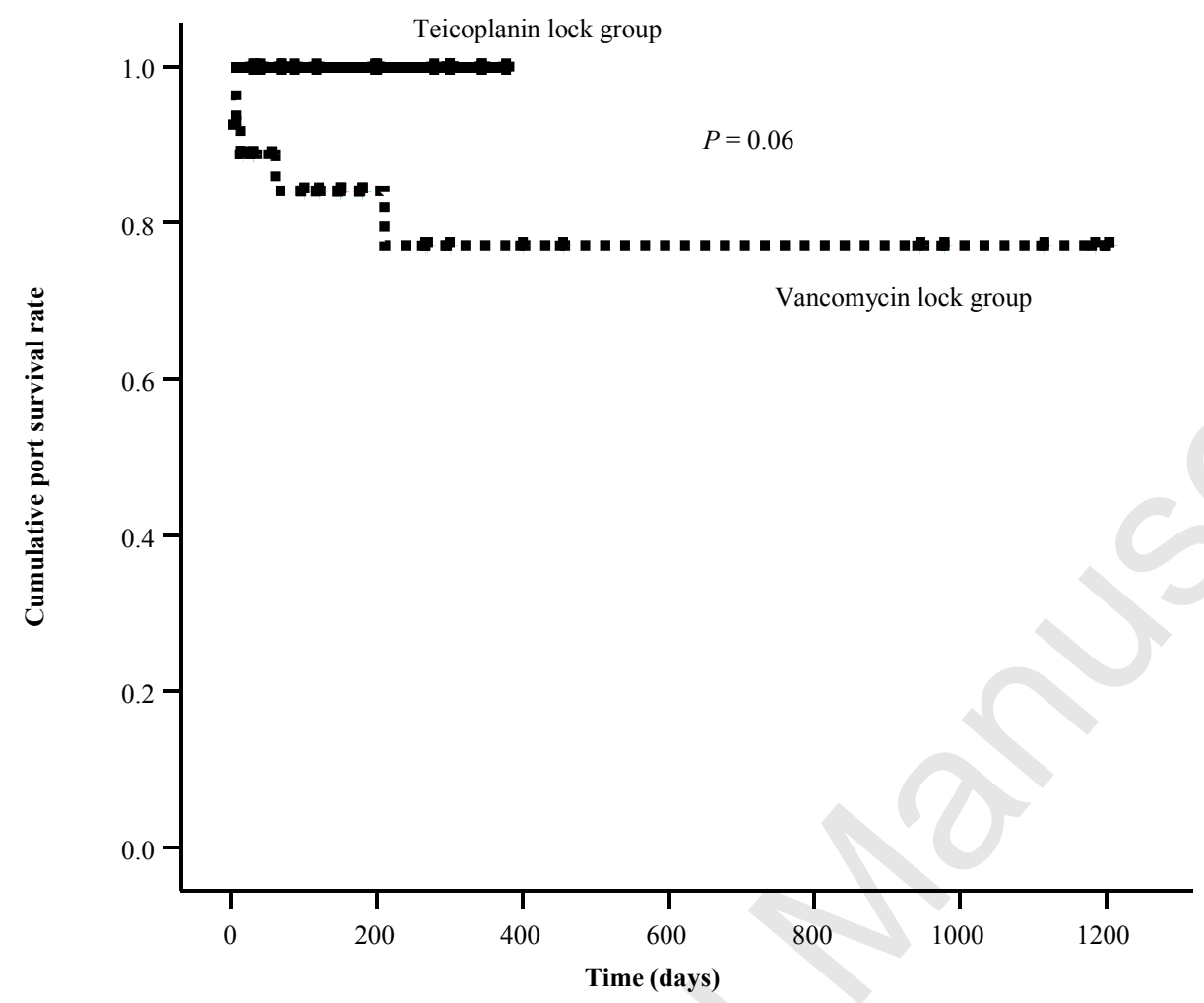

